\title{
Multiple-source optical diffusion approximation for a multilayer scattering medium
}

\author{
Joseph L. Hollmann ${ }^{1}$ and Lihong V. Wang ${ }^{1,2, *}$ \\ 1Optical Imaging Laboratory, Department of Biomedical Engineering, Texas A\&M University, 3120 TAMU, \\ College Station, Texas 77843-3120, USA \\ 2Department of Biomedical Engineering, Washington University in St. Louis, Campus Box 1097, \\ One Brookings Drive, St. Louis, Missouri 63130-4899, USA \\ ${ }^{*}$ Corresponding author: Ihwang@biomed.wustl.edu
}

Received 12 December 2006; revised 24 April 2007; accepted 17 May 2007; posted 22 May 2007 (Doc. ID 78010); published 9 August 2007

\begin{abstract}
A method for improving the accuracy of the optical diffusion theory for a multilayer scattering medium is presented. An infinitesimally narrow incident light beam is replaced by multiple isotropic point sources of different strengths that are placed in the scattering medium along the incident beam. The multiple sources are then used to develop a multilayer diffusion theory. Diffuse reflectance is then computed using the multilayer diffusion theory and compared with accurate data computed by the Monte Carlo method. This multisource method is found to be significantly more accurate than the previous single-source method. (C) 2007 Optical Society of America
\end{abstract}

OCIS codes: $170.3660,290.7050$.

\section{Introduction}

It is well established that the optical properties of biological tissue can reveal underlying physiological parameters [1]. For example, dermatologists have acknowledged the importance of optical absorption in analyzing skin lesions by including color in the ABCD method for skin cancer diagnosis [2]. Garcia-Uribe et al. [3] have shown that quantitatively determining these properties can lead to successful classification of cancerous basal and squamous cell carcinomas from suspicious lesions identified by dermatologists. Knowledge of optical properties is also useful in therapeutic procedures, such as port wine stain and tattoo removals.

Several authors have used the diffusion approximation to the radiative transport equation to determine the optical properties of tissue. Farrell et al. [4] developed a model that predicts light diffuse reflectance from tissue due to a normally incident, infinitesimally narrow light beam. Initially, tissue was approximated as a semi-infinite homogeneous turbid

0003-6935/07/236004-06\$15.00/0

(C) 2007 Optical Society of America medium. However, many tissues, such as skin, cannot be described accurately this way due to their layered structure since a homogeneous model averages the contribution of each layer's optical properties to the diffuse signal. Farrell et al. [5] have shown that tissue cannot be modeled as a single homogeneous layer if the optical properties vary greatly between the layers. Progress has been made toward developing an accurate, multilayer diffusion model. Kienle et al. [6] developed a solution to the diffusion equation for a two-layer turbid medium by replacing a normally incident, infinitesimally narrow light source with an equivalent isotropic point source within the first layer of the medium. However, placing an isotropic point source within the first layer requires the layer boundary to be greater than one transport mean-free path deep. Next, we will illustrate the shortcomings of this approach and propose an improvement that allows for an equivalent isotropic point source inside each layer.

Below, a method for improving the accuracy of the optical diffusion theory for a multilayer scattering medium is presented. The new method will be verified utilizing Monte Carlo simulations. 


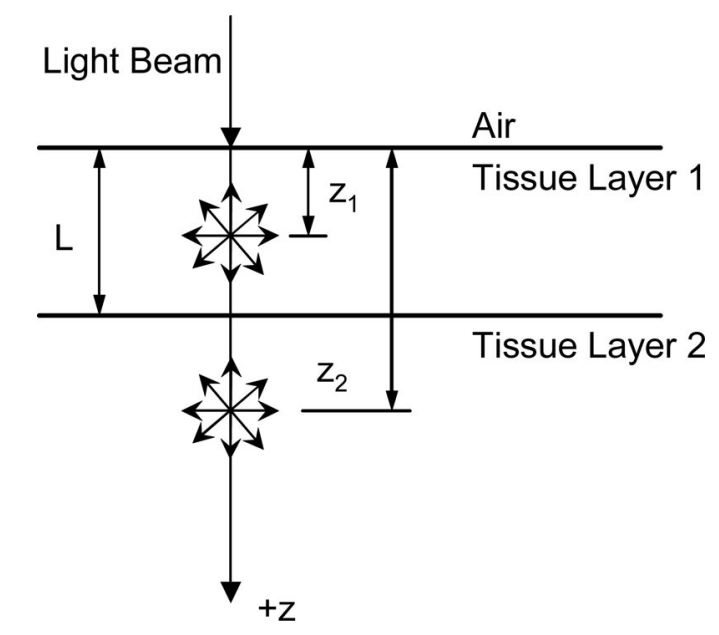

Fig. 1. Positions of isotropic point sources in a two-layer medium. The first layer has a thickness of $L$.

\section{Theory}

\section{A. Geometry}

The problem of interest has an infinitesimally narrow, collimated light beam that is incident perpendicularly on the surface of a two-layer scattering medium (Fig. 1). The coordinate system is set up so that the origin is located at the point of incidence; its $z$ axis points down. The incident beam is replaced by an equivalent isotropic point source in each layer of the turbid medium (to be discussed next). The ambient and turbid media are assumed to have matched indices of reflection.

\section{B. Semi-Infinite Medium}

Light transport through tissue can be described using the absorption coefficient $\left(\mu_{a}\right)$, the scattering coefficient $\left(\mu_{s}\right)$, and the anisotropy factor $(g)$. The coefficients, $\mu_{a}$ and $\mu_{s}$, describe the probabilities of absorption and scattering per unit infinitesimal length, respectively. The anisotropy factor, $g$, is the cosine of the average scattering polar angle. Most biological tissue is highly forward scattering $(g>0.8)$. The scattering coefficient of the medium can be replaced by an equivalent transport or reduced scattering coefficient given as $\mu_{s}{ }^{\prime}=\mu_{s}(1-g)$ in the diffusive regime [4,7].

In general, tissue has a high reduced scattering coefficient and a low absorption coefficient, which means a photon is likely to undergo many scattering events before absorption or reemission. For this reason, the diffusion approximation to the radiative transport equation has commonly been adopted.

Another approximation states that the diffuse reflectance from an infinitesimally narrow, collimated beam incident on the surface of a turbid medium can be represented by that from an equivalent isotropic point source buried within the medium. The diffusion equation for a semi-infinite homogeneous medium is

$$
\nabla^{2} \Phi(x, y, z)-\frac{\mu_{a}}{D} \Phi(x, y, z)=-\frac{w}{D} \delta\left(x, y, z-z_{0}\right),
$$

where $\Phi$ is the fluence rate, $w$ is the strength of the equivalent point source, and $D=\left[3\left(\mu_{s}{ }^{\prime}+\mu_{a}\right)\right]^{-1}$ is the diffusion constant. The Dirac delta function located at $z_{0}$ is the position of the equivalent point source within the medium.

The location and strength of the equivalent point source is given by the optical properties of the medium. A photon that enters the medium along the $z$ direction will have an exponentially decaying probability of progressing to depth $z$ before being either scattered or absorbed. Each first interaction site in the equivalent isotropically scattering medium produces an isotropic point source with a strength equal to the transport scattering albedo, given by $a^{\prime}=$ $\mu_{s}^{\prime} /\left(\mu_{a}+\mu_{s}^{\prime}\right)$. A single isotropic source is estimated by the probability-weighted mean of the positions of the first interactions in the equivalent medium. For a semi-infinite medium the position is given by the transport mean-free path

$$
L_{t}^{\prime}=\frac{1}{\mu_{t}^{\prime}},
$$

where $\mu_{t}{ }^{\prime}$ is the transport interaction coefficient, defined as $\mu_{t}{ }^{\prime}=\mu_{a}+\mu_{s}{ }^{\prime}$. The strength of the equivalent source is equal to $a^{\prime}$. The accuracy of the diffusion equation is limited to a region of "far diffuse reflectance," [8] generally considered to be greater than one or two transport mean-free paths away from the source, depending on the desired accuracy $[4,8,9]$.

\section{Two-Layer Medium}

For a two-layer turbid medium, Fig. 1, each layer has independent reduced scattering and absorption coefficients. The thickness of the first layer is $L$. The boundary between the turbid and the ambient media is treated with the extrapolated boundary condition [4]. We model the fluence rate in the medium and the diffuse reflectance by placing an equivalent source in each layer. These equivalent sources, rather than the incident collimated beam, are used for further computation.

The diffusion equation for a two-layer medium is given as

$$
\begin{gathered}
\nabla^{2} \Phi_{i}(x, y, z)-\frac{\mu_{a i}}{D_{i}} \Phi_{i}(x, y, z)=\frac{-w_{i}}{D_{i}} \delta\left(x, y, z-z_{i}\right) \\
i=1,2
\end{gathered}
$$

where $z_{i}$ corresponds to the isotropic point source position in the $i$ th layer. The equivalent source positions are given by the probability-weighted mean of the first-scattering locations along the $z$ axis within 
each layer:

$$
\begin{aligned}
& z_{1}=\frac{\int_{0}^{L} z \exp \left(-\mu_{t 1}{ }^{\prime} z\right) d z}{\int_{0}^{L} \exp \left(-\mu_{t 1}{ }^{\prime} z\right) d z}=\frac{1-\left(\mu_{t 1}{ }^{\prime} L+1\right) \exp \left(-\mu_{t 1}{ }^{\prime} L\right)}{\mu_{t 1}{ }^{\prime}\left(1-\exp \left(-\mu_{t 1}{ }^{\prime} L\right)\right)}, \\
& z_{2}=\frac{\int_{L}^{\infty} z \exp \left(-\mu_{t 1}{ }^{\prime} L-\mu_{t 2}{ }^{\prime}(z-L)\right) d z}{\int_{L}^{\infty} \exp \left(-\mu_{t 1}{ }^{\prime} L-\mu_{t 2}{ }^{\prime}(z-L)\right) d z}=L+\frac{1}{\mu_{t 2}{ }^{\prime}} .
\end{aligned}
$$

Each equivalent source is given a strength (or weighting):

$$
\begin{aligned}
w_{1}(z) & =\mu_{s 1}{ }^{\prime} \int_{0}^{L} \exp \left(-\mu_{t 1}{ }^{\prime} z\right) d z=a_{1}{ }^{\prime}\left(1-\exp \left(-\mu_{t 1}{ }^{\prime} L\right)\right) \\
w_{2}(z) & =\mu_{s 2}{ }^{\prime} \int_{L}^{\infty} \exp \left(-\mu_{t 1}{ }^{\prime} L-\mu_{t 2}{ }^{\prime}(z-L)\right) d z \\
& =a_{2}{ }^{\prime} \exp \left(-\mu_{t 1}{ }^{\prime} L\right) .
\end{aligned}
$$

These equations are reduced to the semi-infinite case by setting $L$ to zero or infinity.

As mentioned above, a two-layer model for light propagation through tissue was previously developed. This model utilizes a single, equivalent, and isotropic point source with a strength and location based on the first layer's optical properties [6]. This method would fail when the first interaction sites in the second layer cannot be neglected.

\section{Solution}

The solution of the two-layer diffusion equations is given below. The first step in solving Eq. (3) is to take two-dimensional Fourier transformation in the $x-y$ space:

$$
\begin{gathered}
\frac{\partial^{2}}{\partial z^{2}} \phi_{i}\left(z, s_{a}, s_{b}\right)-\alpha_{i}^{2} \phi_{i}\left(z, s_{a}, s_{b}\right)=-\frac{w_{i}}{D_{i}} \delta\left(z-z_{i}\right), \\
i=1,2,
\end{gathered}
$$

where $s_{a}$ and $s_{b}$ denote spatial-frequency dummy variables and $\phi_{i}$ denotes the Fourier transform of $\Phi_{i}$. We can take advantage of the radial homogeneity by saying that $s^{2}=s_{a}{ }^{2}+s_{b}{ }^{2}$ and by introducing variable $\alpha_{i}{ }^{2}=\left(D_{i} s^{2}+\mu_{a i}\right) / D_{i}=s^{2}+\mu_{\text {eff } i}{ }^{2}$, where the effective attenuation coefficient $\mu_{\text {eff } i}=\sqrt{\mu_{a i} / D_{i}}$.

To solve Eq. (6) we use the extrapolated boundary condition to deal with the interface between the ambient and the scattering media [10]. This gives us a plane at position $z=-z_{b}$, where the fluence rate is approximately zero, i.e.,

$$
\phi_{1}\left(-z_{b}, s\right)=0 \text {, }
$$

where $z_{b}=2 D_{1}$ for a refractive-index matched boundary. Since the second layer is infinitely deep, we have

$$
\phi_{2}(+\infty, s)=0 .
$$

Assuming a uniform index of refraction in the tissue, we have the following continuity of fluence rate and flux at the boundary between the layers:

$$
\begin{gathered}
\frac{\phi_{1}(L, s)}{\phi_{2}(L, s)}=1, \\
\left.D_{1} \frac{\partial \phi_{1}(z, s)}{\partial z}\right|_{z=L}=\left.D_{2} \frac{\partial \phi_{2}(z, s)}{\partial z}\right|_{z=L} .
\end{gathered}
$$

Solving the differential equations with the boundary conditions above, we obtain

$$
\left.\begin{array}{rl}
\phi_{1}(z, s)= & w_{1} / 2 \alpha_{1} D_{1} \\
& {\left[\begin{array}{l}
\frac{\left(\alpha_{2} D_{2}-\alpha_{1} D_{1}\right)}{\left(\alpha_{2} D_{2}+\alpha_{1} D_{1}\right) \exp \left(\alpha_{1}\left(z+2 L+2 z_{b}-z_{1}\right)\right)+\left(\alpha_{1} D_{1}-\alpha_{2} D_{2}\right) \exp \left(\alpha_{1}\left(z-z_{1}\right)\right)}+\exp \left(-\alpha_{1}\left|z-z_{1}\right|\right) \\
-\frac{\left(\alpha_{1} D_{1}+\alpha_{2} D_{2}\right)}{\left(\alpha_{1} D_{1}+\alpha_{2} D_{2}\right) \exp \left(\alpha_{1}\left(z+z_{1}+2 z_{b}\right)\right)+\left(\alpha_{1} D_{1}-\alpha_{2} D_{2}\right) \exp \left(\alpha_{1}\left(z+z_{1}-2 L\right)\right)} \\
+\frac{\left(\alpha_{1} D_{1}-\alpha_{2} D_{2}\right)}{\left(\alpha_{1} D_{1}+\alpha_{2} D_{2}\right) \exp \left(\alpha_{1}\left(2 L-z_{1}-z\right)\right)+\left(\alpha_{1} D_{1}-\alpha_{2} D_{2}\right) \exp \left(-\alpha_{1}\left(z+z_{1}+2 z_{b}\right)\right)} \\
+\frac{\left(\alpha_{2} D_{2}-\alpha_{1} D_{1}\right)}{\left(\alpha_{1} D_{1}+\alpha_{2} D_{2}\right) \exp \left(\alpha_{1}\left(z_{1}+2 z_{b}+2 L-z\right)\right)+\left(\alpha_{1} D_{1}-\alpha_{2} D_{2}\right) \exp \left(\alpha_{1}\left(z_{1}-z\right)\right)}
\end{array}\right], \quad z \leq L,}
\end{array}\right],
$$




$$
\begin{aligned}
\phi_{2}(z, s)= & \frac{\left(\alpha_{2} D_{2} w_{1} \exp \left(-\alpha_{1}\left(L-z_{1}\right)\right)-\alpha_{1} D_{1} w_{2} \exp \left(-\alpha_{2}\left(z_{2}-L\right)\right)\right)}{2\left(\alpha_{2} D_{2} \tanh \left(\alpha_{1}\left(z_{b}+L\right)\right)+\alpha_{1} D_{1}\right)} \frac{\exp \left(-\alpha_{2}(z-L)\right)}{\alpha_{2} D_{2}} \\
& +\frac{\left(w_{2} \exp \left(-\alpha_{2}\left(z_{2}-L\right)\right)+w_{1} \exp \left(-\alpha_{1}\left(L-z_{1}\right)\right)\right) \sinh \left(\alpha_{1}\left(L+z_{b}\right)\right)-w_{1} \exp \left(-\alpha_{1}\left(z_{b}+z_{1}\right)\right)}{2\left(\alpha_{2} D_{2} \sinh \left(\alpha_{1}\left(z_{b}+L\right)\right)+\alpha_{1} D_{1} \cosh \left(\alpha_{1}\left(z_{b}+L\right)\right)\right)} \\
& \times \exp \left(-\alpha_{2}(z-L)\right)+\frac{w_{2} \exp \left(-\alpha_{2}\left|z_{2}-z\right|\right)}{2 \alpha_{2} D_{2}}, \quad z>L .
\end{aligned}
$$

To solve for the fluence rate, we must use the following inverse two-dimensional Fourier transformation of $\phi_{i}$ :

$$
\begin{aligned}
\Phi_{i}(x, y, z)= & \frac{1}{4 \pi^{2}} \int_{-\infty}^{\infty} \int_{-\infty}^{\infty} \phi_{i}(z, s) \\
& \times \exp \left(-i\left(s_{a} x+s_{b} y\right)\right) d s_{a} d s_{b} .
\end{aligned}
$$

From the radial symmetry, we obtain the following equation in cylindrical coordinates:

$$
\Phi_{i}(\rho, z)=\frac{1}{2 \pi} \int_{0}^{\infty} s \phi_{i}(z, s) J_{0}(s \rho) d s
$$

where $J_{0}$ is the Bessel function of the first kind of the zeroth order, and $\rho^{2}=x^{2}+y^{2}$. This integration is solved numerically using Simpson's integration formula [11]. The fluence rate is then used to solve for the spatially resolved diffuse reflectance as follows:

$$
R(\rho)=\frac{1}{4} \Phi_{1}(\rho, z=0)+\left.\frac{1}{2} D_{1} \frac{\partial \Phi_{1}(\rho, z)}{\partial z}\right|_{z=0} .
$$

\section{Simulation}

The above solution to the two-layer diffusion equation is programmed and verified with Monte Carlo simulation results, which are used as a "gold" standard $[12,13]$. The reflectance was simulated for radial distances up to $1 \mathrm{~cm}$ from the incident photon beam. For each simulation $10 \times 10^{6}$ photons were traced.

Both the single-source and the double-source approximations are tested for a two-layer medium with a first-layer thickness greater than one transport mean-free path $\left(L>1 / \mu_{t 1}{ }^{\prime}\right)$. In other words, the first layer is optically thick. In this case, we can test the single-source solution against the two-source solution. The parameters used are $\mu_{s 1}=90 \mathrm{~cm}^{-1}, \mu_{a 1}=$ $0.02 \mathrm{~cm}^{-1}, \mu_{\mathrm{s} 2}=110 \mathrm{~cm}^{-1}, \mu_{a 2}=0.1 \mathrm{~cm}^{-1}, \mathrm{~g}=0.9$, and $L=0.12 \mathrm{~cm}$. The index of refraction is matched across the two layers and ambient medium.

Figure 2(a) shows the diffuse reflectance for distances up to $1 \mathrm{~cm}$ from the source. We see good agreement between the Monte Carlo results and both diffusion models. However, if we compare the relative error between the diffusion models and the Monte Carlo method Fig. 2(b), we see that the two-source model has a smaller relative error than the singlesource model.

The reduced scattering coefficient is derived utilizing the similarity relation between scattering in an anisotropic medium and scattering in an isotropic medium [7]. The relationship is central to the derivation of the two-layer diffusion equation. To test the validity of the diffusion equation for two layers, the Monte Carlo simulations were programmed for $g=$ 0.5 and $g=0$. The reduced scattering and absorption

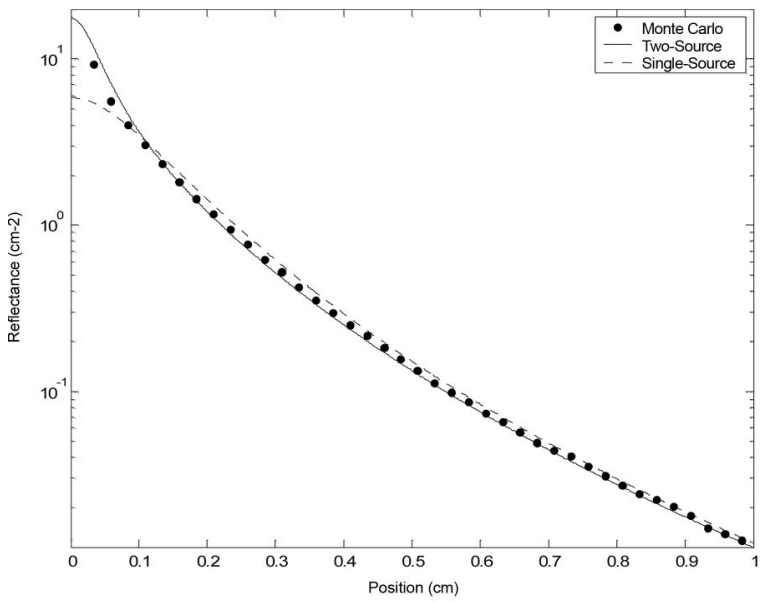

(a)

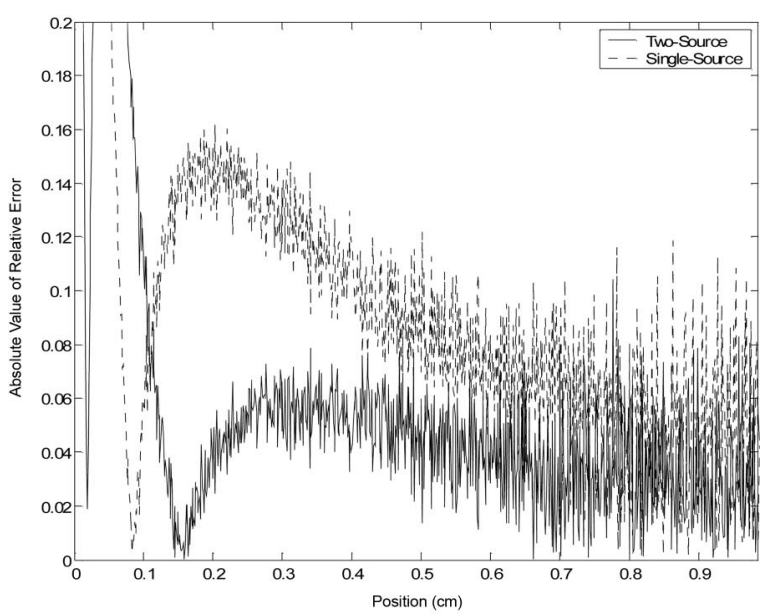

(b)

Fig. 2. (a) Comparison of diffuse reflectance calculated with single-source (dotted line), double-source approximations (solid line) and with the Monte Carlo method (circles). (b) Absolute value of the relative error for the single-source (dotted line) and doublesource (solid line) diffusion approximations. 
coefficients were kept the same as above for each layer. The scattering coefficients were solved for as follows:

$$
\mu_{s i}=\frac{\mu_{s i}{ }^{\prime}}{1-g_{i}}
$$

where the subscript $i$ refers to either layer 1 or 2 .

The absolute value of the error between the two diffusion solutions and the Monte Carlo results for $g=0.5$ are shown in Fig. 3(a). The relative error for the two-source solution falls below $2 \%$ for distances greater than $2 \mathrm{~mm}$. The single-source solution trends toward $6 \%$ error at large distances.

Figure 3(b) shows the relative error between the two diffusion solutions and the Monte Carlo results for $g=0$. The two-source solution falls below $10 \%$ error for distances greater than $0.5 \mathrm{~cm}$. However, the

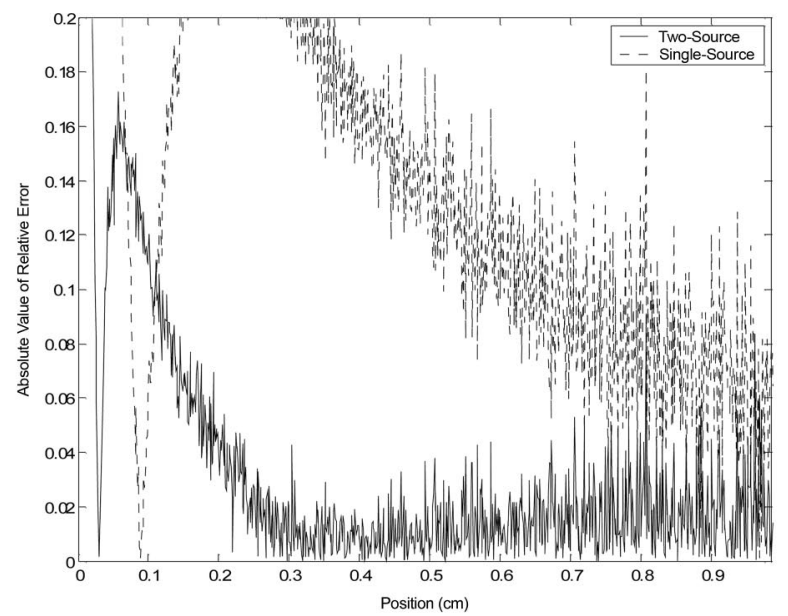

(a)

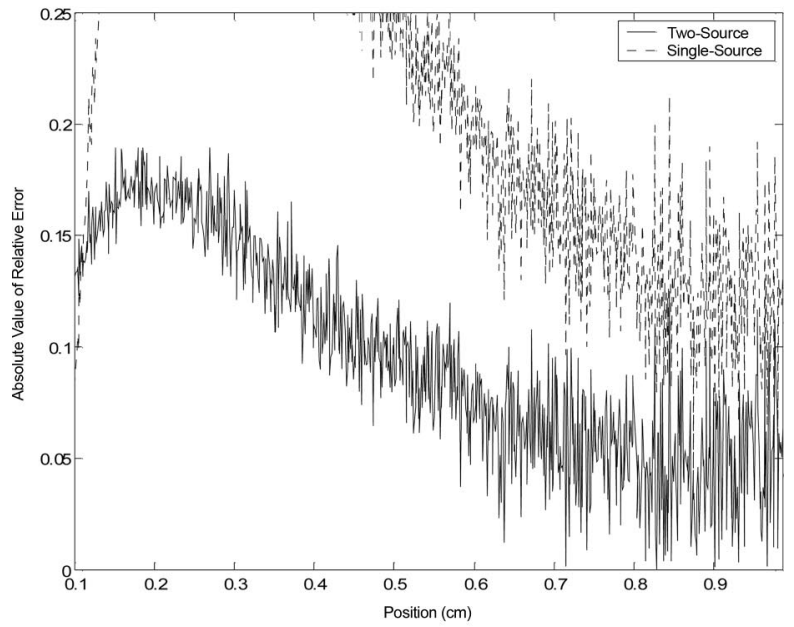

(b)

Fig. 3. (a) Absolute value of the relative error for the singlesource (dotted line) and double-source (solid line) diffusion approximations for $g=0.5$. (b) Absolute value of the relative error for the single-source (dotted line) and double-source (solid line) diffusion approximations for $g=0$. The reduced scattering and absorption coefficients are the same as those used for Fig. 2. single-source solution appears to trend above a relative error of $10 \%$ for up to $1 \mathrm{~cm}$.

The next simulation was created to test the validity of the two-source diffusion equation with a top layer thickness of less than one transport mean-free path $\left(L<1 / \mu_{t 1}{ }^{\prime}\right)$. In other words, the first layer is considered optically thin. In this case, the single-source model is not applicable but the two-source model still applies since it is not limited by the thickness of the first layer as is the single-source model. The parameters utilized for the two-layer Monte Carlo simulation are $\mu_{s 1}=110 \mathrm{~cm}^{-1}, \mu_{a 1}=0.06 \mathrm{~cm}^{-1}, \mu_{s 2}=$ $90 \mathrm{~cm}^{-1}, \mu_{a 2}=0.1 \mathrm{~cm}^{-1}, g=0.9$, and $L=0.06 \mathrm{~cm}$. As above, the index of refraction is matched across the two layers and ambient medium. The results in Fig. 4(a) show that the two-source solution closely follows the Monte Carlo results.

The necessity of utilizing a multiple-layer diffusion model is not clear when the first layer of a two-layer

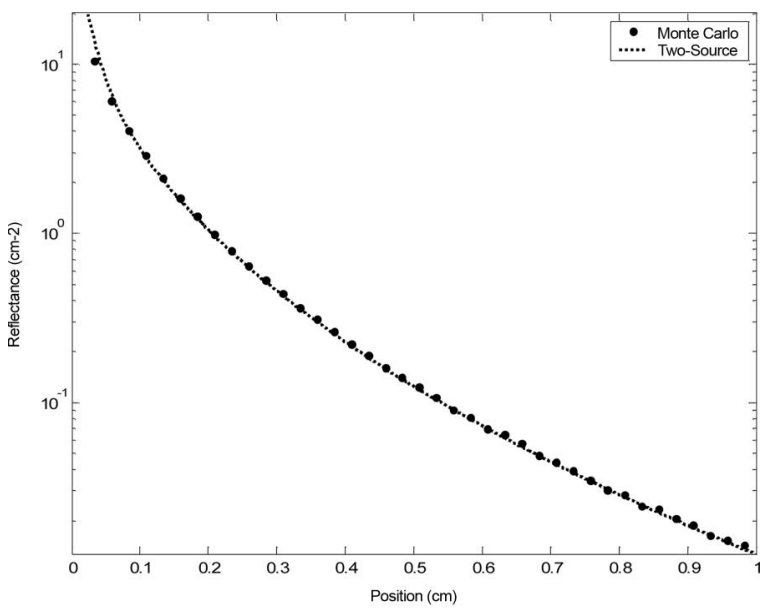

(a)

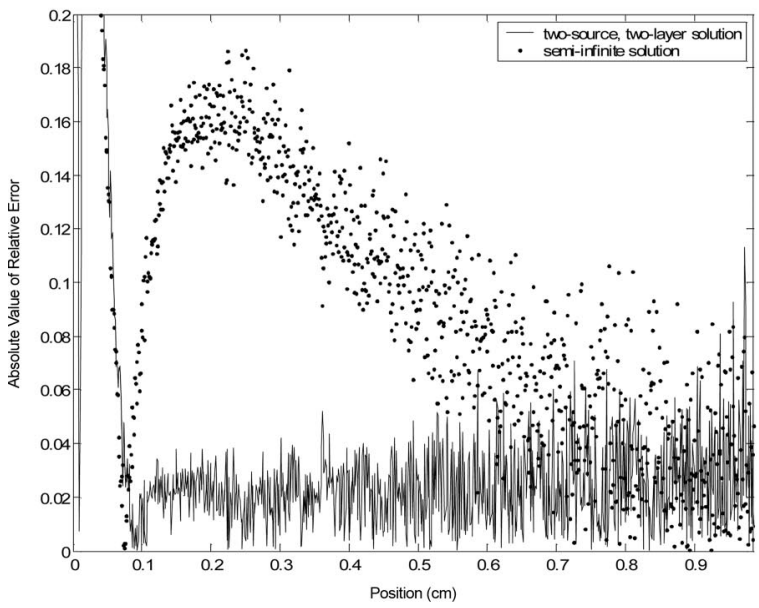

(b)

Fig. 4. (a) Comparison of diffuse reflectance calculated with the double-source approximation (solid line) and with the Monte Carlo method (circles). The first layer is optically thin. (b) Relative error for the double-source diffusion approximation (line) and the semiinfinite diffusion equation solution utilizing the optical properties of the second layer (dots). 
medium is optically thin. This is because the first layer may not have a large effect on the reflected light. To test the utility of the two-source equation for an optically thin layer, the diffusion solution for a semi-infinite homogeneous medium was also solved for utilizing the optical properties of the second layer. The effect of the first layer on the reflectance is ignored. The optical properties are $\mu_{s}=90 \mathrm{~cm}^{-1}, \mu_{a}$ $=0.1 \mathrm{~cm}^{-1}$, and $g=0.9$. Figure $4(\mathrm{~b})$ shows that the two-source solution has a relative error of less than $4 \%$ after $1 \mathrm{~mm}$ while the semi-infinite solution has a maximum error of $16 \%$ at $2 \mathrm{~mm}$. It is not clear if the semi-infinite solution will converge to the Monte Carlo results if the distances were extended beyond $1 \mathrm{~cm}$.

\section{Conclusions}

We have introduced a new method for solving for the diffuse reflectance from a multilayer turbid medium. Our study indicates that the fluence rate inside a multilayer turbid medium can best be modeled by multiple, equivalent, and isotropic point sources. Based on the validations with Monte Carlo simulations, the two-source approximation performs better than the single-source approximation. The two-source approximation is also accurate for an optically thin first layer, whereas the single-source solution cannot be applied. It can be shown from Eq. (5) that, as the thickness of layer 1 approaches zero, so does the strength of the source embedded within its medium. Conversely, as the thickness of layer 1 grows toward infinity, the strength of the source embedded within the second layer also approaches zero. Therefore, we can see that the two-source solution is valid for any thickness of layer 1. By removing this dependency, we have created a much more robust solution for the diffusion equation for a two-layer turbid medium. The improved accuracy from utilizing the two-source diffusion solution for an optically thin first layer is illustrated in Fig. 4. The solution can be extended to more than two layers.

We thank A. Garcia-Uribe for checking the derivations. This work was supported by the National Institutes of Health grant R01 CA106728.

\section{References}

1. R. Richards-Kortum and E. Sevick-Muraca, "Quantitative optical spectroscopy for tissue diagnosis," Annu. Rev. Phys. Chem. 555-606 (1996).

2. A. Bono, S. Tomatis, and C. Bartoli, et al., "The ABCD system of melanoma detection: A spectrophotometric analysis of the asymmetry, border, color, and dimension," Cancer 85, 72-77 (1999).

3. A. Garcia-Uribe, N. Kehtarnavaz, G. Marquez, V. Prieto, M. Duvic, and L. H. Wang, "Skin cancer detection using spectroscopic oblique-incidence reflectometry: classification and physiological origins," Appl. Opt. 43, 2643-2650 (2004).

4. T. J. Farrell, M. S. Patterson, and B. Wilson, "A diffusion theory model of spatially resolved, steady-state diffuse reflectance for the noninvasive determination of tissue optical properties in vivo," Med. Phys. 19, 879-888 (1992).

5. T. J. Farrell, M. S. Patterson, and M. Essenpreis, "Influence of layered tissue architecture on estimates of tissue optical properties obtained from spatially resolved diffuse reflectometery," Appl. Opt. 37, 1958-1972 (1998).

6. A. Kienle, M. S. Patterson, N. Dognitz, R. Bays, G. Wagnieres, and H. Van Den Bergh, "Noninvasive determination of the optical properties of two-layered turbid media," Appl. Opt. 37, 779-791 (1998).

7. G. Yoon, S. A. Prahl, and A. J. Welch, "Accuracies of the diffusion approximation and its similarity relations for laser irradiated biological media," Appl. Opt. 28, 2250-2255 (1989).

8. G. Marquez and L.-H. Wang, "White light oblique incidence reflectometer for measuring absorption and reduced scattering spectra of tissue-like turbid media," Opt. Express 1, 454-460 (1997).

9. L.-H. Wang and S. L. Jacques, "Source of error in calculation of optical diffuse reflectance from turbid media using diffusion theory," Computer Methods and Programs in Biomedicine 61, 163-170 (2000).

10. R. C. Haskell, L. O. Svaasand, T. T. Tsay, T. C. Feng, M. McAdams, and B. J. Tromberg, "Boundary conditions for the diffusion equation in radiative transfer," J. Opt. Soc. Am. A 11, 2727-2741 (1994).

11. M. N. O. Sadiku, "Finite difference methods," in Numerical Techniques in Electromagnetics, 2nd Ed. (CRC Press, 2001), pp. 197-199.

12. L.-H. Wang, S. L. Jacques, and L.-Q. Zheng, "MCML-Monte Carlo modeling of photon transport in multi-layered tissues," Comput. Methods Programs Biomed. 47, 131-146 (1995).

13. S. T. Flock, B. C. Wilson, and M. S. Patterson, "Monte Carlo modeling of light propagation in highly scattering tissues-II: comparison with measurements in phantoms," IEEE Trans. Biomed. Eng. 36, 1169-1173 (1989). 